DOI: $10.1515 / \mathrm{rrlm}-2017-0028$

\title{
Effects of low-molecular weight alcohols on bacterial viability
}

\author{
Adrian Man ${ }^{1}$, Andrei Şerban Gâz ${ }^{2 *}$, Anca Delia Mare ${ }^{1}$, Lavinia Berţa ${ }^{3}$ \\ 1. Department of Microbiology, Faculty of Medicine, University of Medicine and Pharmacy of Tîrgu \\ Mureș, Romania \\ 2. Department of Organic Chemistry, Faculty of Pharmacy, University of Medicine and Pharmacy of \\ Tîrgu Mureș, Romania \\ 3. Department of General and Inorganic Chemistry, Faculty of Pharmacy, University of Medicine and \\ Pharmacy of Tîrgu Mureș, Romania
}

\begin{abstract}
Alcohol based solutions are among the most convenient and wide spread aid in the prevention of nosocomial infections. The current study followed the efficacy of several types and isomers of alcohols on different bacterial species. Seven alcohols (ethyl, n-propyl, iso-propyl, n-butyl, iso-butyl, tert-butyl alcohol, and ethylene glycol) were used to evaluate their minimal inhibitory and bactericidal effects by microdilution method on bacteria that express many phenotypical characteristics: different cell-wall structure (Gram positive/negative bacteria), capsule production (Klebsiella pneumoniae), antibiotic resistance (MRSA vs MSSA) or high environmental adaptability (Pseudomonas aeruginosa). Results: The best inhibitory effect was noticed for n-propyl, followed by iso-propyl, n-butyl, and iso-butyl alcohols with equal values. Ethylene glycol was the most inefficient alcohol on all bacteria. In K. pneumoniae and P. aeruginosa, the bactericidal concentrations were higher than the inhibitory one, and to a level similar to that encountered for most of the Gram-positive bacteria. Among Gram-positive cocci, E. faecalis presented the lowest susceptibility to alcohols. Conclusions: All alcohols presented good effect on bacteria, even in low concentrations. Compared to ethanol as standard, there are better alternatives that can be used as antimicrobials, namely longer-chain alcohols such as propyl or butyric alcohols and their iso-isomers. Ethylene glycol should be avoided, due to its toxicity hazard and low antimicrobial efficacy. Bacterial phenotype (highly adaptable bacteria, biofilm formation) and structure (cell wall structure, presence of capsule) may drastically affect the responsiveness to the antimicrobial activity of alcohols, leading to higher bactericidal than inhibitory concentrations.
\end{abstract}

Keywords: antimicrobial activity, bacterial resistance, alcohols, isomers, disinfectant and antiseptic

Received: 22 $2^{\text {nd }}$ March 2017; Accepted: 12 ${ }^{\text {th }}$ June 2017; Published: $24^{\text {th }}$ August 2017

*Corresponding author: Andrei Serban Gaz Florea, University of Medicine and Pharmacy of Tirgu Mures, Tirgu Mures, Romania. E-mail: andrei.gaz@umftgm.ro 


\section{Introduction}

Health care associated infections are nowadays of great interest for patients, medical staff, insurance companies, and regulatory divisions. Usually, healthcare personnel represent the main route of nosocomial infection transmission, making antiseptics and disinfectants imperative to be used in hospitals and other health care facilities. These biocides are used on topical or hard-surface applications and are part of infection control and prevention of nosocomial infections (1-3), but only if used in corresponding concentrations end exposure time. There is no doubt that a good hand hygiene reduces the risk of cross-contaminations and the spreading of nosocomial infections (4). Among the disinfectants (e.g. aldehyde, halogen-releasing agents, phenols), alcohols are the most used due to their good effectiveness, low cost and availability, especially in less developed countries $(5,6)$. Although several alcohols have been considered to have antimicrobial effects, only three of them are widely used, namely ethyl alcohol - ethanol, isopropyl alcohol - isopropanol (or propan-2-ol), and $n$-propyl alcohol - $n$-propanol (or propan-1-ol) (7).

Up to date alcohol based solutions with ethanol as the main ingredient are among the most convenient and widespread aid in the prevention of nosocomial infections (8). Better efficacy has proven for either isopropyl alcohol alone or mixed with ethanol in some formulation, used especially in the USA, however, some controversy still exists (9). Lately, 1-propanol was introduced as a better alternative to ethanol as disinfectant formula in some European countries.

Most studies focused on the immediate effect of the alcohols on bacteria and the development of new antibacterial compounds based on alcohol (short exposure-high concentrations). However, not much is known about the repeated and prolonged exposure of bacteria to alcohol solutions or about bacterial adaptability in such conditions. Thus, the present study followed the effects of bacteria exposure to alcohols using a methodology that is based on quantitative antimicrobial susceptibility testing (microdilution). The specific aim was to study the efficacy of small concentrations of several types and isomers of alcohols on different bacterial species and to compare the results with those for ethanol, considered as a standard.

\section{Materials and methods}

\section{Alcohols}

To determine the minimum inhibitory/bactericidal concentrations on Gram-positive and Gram-negative bacterial strains, seven alcohols have been used: ethyl alcohol (EA), n-propyl alcohol ( $n \mathrm{PA})$, iso-propyl alcohol (iPA), $n$-butyl alcohol ( $n \mathrm{BA})$, iso-butyl alcohol ( $i \mathrm{BA})$, tert-butyl alcohol ( $t \mathrm{BA})$, and ethylene glycol (EG) (Table 1). All the alcohols were of analytical grade from Merck (Germany) or Sigma Aldrich and were used without any further purification.

\section{Bacterial strains}

Gram-positive bacteria (methicillin sensitive Staphylococcus aureus - MSSA ATCC 29213, methicillin resistant Staphylococcus aureus - MRSA ATCC 43300, Enterococcus faecalis ATCC 29212) and Gram-negative bacteria (Escherichia coli ATCC 25922, Klebsiella pneumoniae ATCC 13883, Pseudomonas aeruginosa ATCC 27853) used in this study were part of the collection of the Department of Microbiology, University of Medicine and Pharmacy of Tîrgu Mureș. All bacterial strains were maintained in Tryptic Soy Broth (TSB) medium with glycerin, at $-70^{\circ} \mathrm{C}$. Forty-eight hours before the study, the strains were revitalized on Columbia blood agar and then repassed to obtain a fresh, pure culture. 
Table 1 - Structure of the used alcohols

\begin{tabular}{|c|c|}
\hline Alcohol name & Alcohol structure \\
\hline Ethyl alcohol & $\mathrm{H}_{3} \mathrm{C}^{-}$ \\
\hline n-Propyl alcohol & $\mathrm{H}_{3} \mathrm{C}$ \\
\hline $\begin{array}{l}\text { iso-Propyl alco- } \\
\text { hol }\end{array}$ & \\
\hline n-Butyl alcohol & $\mathrm{H}_{3} \mathrm{C}^{-}$ \\
\hline iso-Butyl alcohol & $\mathrm{H}_{3} \mathrm{C}$ \\
\hline tert-Butyl alcohol & $\mathbf{H}_{3}$ \\
\hline Ethylene glycol & $\mathrm{OH}$ \\
\hline
\end{tabular}

\section{Determination of MIC}

To determine the minimum inhibitory concentrations (MIC), $200 \mu \mathrm{l}$ of each pure alcohol were pipetted in the first well of rows A-H of a 96-well plate and then diluted with distilled sterile water, using a multichannel pipette. The initial concentration $(\mathrm{V} / \mathrm{V})$ of each alcohol in wells $1-12$ was $100 \%, 75 \%, 50 \%, 25 \%, 12.5 \%, 6.25 \%$, $3.125 \%, 1.562 \%, 0.781 \%, 0.39 \%, 0.195 \%$ and $0.097 \%$.

Microbial inoculum suspensions were prepared from isolated colonies at $0.5 \mathrm{McF}$ arland standard turbidity in sterile saline solution, which is roughly equivalent to 150 million cells per $\mathrm{mL}\left(1.5 \times 10^{8} \mathrm{CFU} / \mathrm{mL}\right)$. From this, 10 $\mu 1$ were transferred to $9990 \mu 12 \mathrm{x}$ concentrated TSB culture medium; one hundred microliters from each bacterial mix were transferred to their corresponding 96-well plate using a multichannel pipette, over the $100 \mu \mathrm{l}$ of alcohol solutions. Thus, the alcohol concentration $(\mathrm{V} / \mathrm{V})$ in the final volume in wells $1-12$ was $50 \%, 37.5 \%$. $25 \%, 12.5 \%, 6.25 \%, 3.125 \%, 1.562 \%, 0.781 \%$, $0.39 \%, 0.195 \%, 0.097 \%$, and $0.048 \%$, each well containing approximatively $1.5 \times 10^{3} \mathrm{CFU}$.

One 96-well plate was prepared for each bacterial strain. Two wells from each plate were used for positive growth control $(100 \mu \mathrm{l}$ of each bacterial inoculum mixed with $100 \mu 1$ sterile water) respectively negative growth control $(100 \mu \mathrm{l}$ TSB $2 \mathrm{x}$ mixed with $100 \mu 1$ sterile water).

All the plates were incubated at $37^{\circ} \mathrm{C}$ for $24 \mathrm{~h}$ in normal atmosphere. The MIC for each alcohol was read in the first well of each row where no bacterial growth was noted (clear culture medium).

\section{Determination of $M B C$}

The minimum bactericidal concentrations (MBC) of each alcohol was assessed by spot-inoculating five microliters on agar plate, from the last three wells that showed no bacterial growth after incubation. The MBC for each alcohol was read in the first of the three spots where no bacterial colonies grew.

\section{Results}

We tested the inhibitory and bactericidal effects of seven alcohols on six bacterial strains that are representative for the most common pathogenic species: methicillin sensitive Staphylococcus aureus, methicillin resistant Staphylococcus aureus, Enterococcus faecalis, Escherichia coli, Klebsiella pneumoniae and Pseudomonas aeruginosa (Figure 1). We chose these particular strains as they express many different phenotypical characteristics: different cell-wall structure (Gram positive/negative bacteria), capsule production ( $K$. pneumoniae), antibiotic 


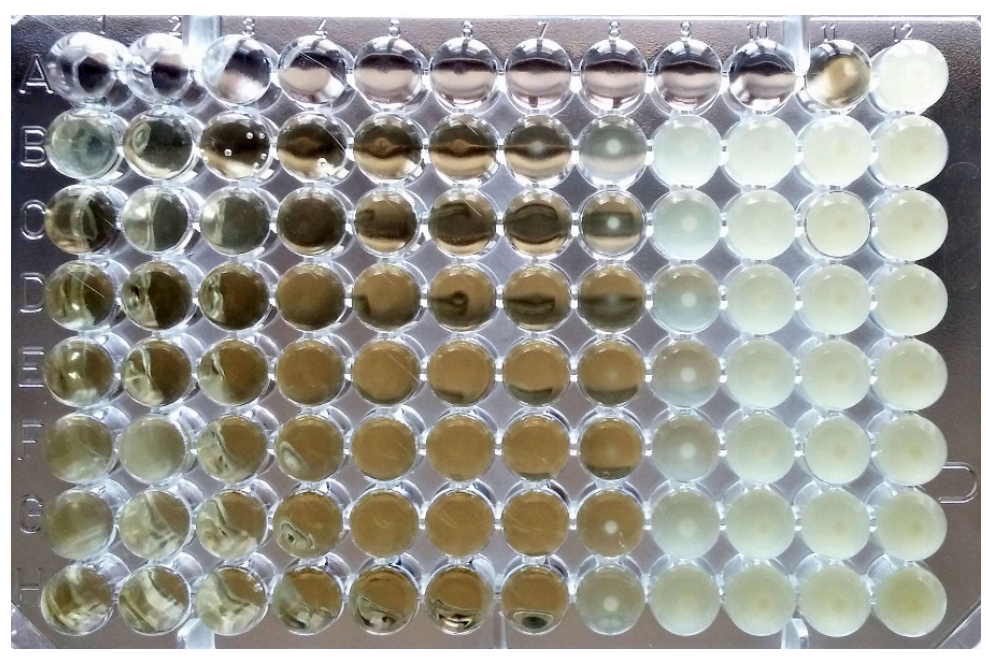

1.A

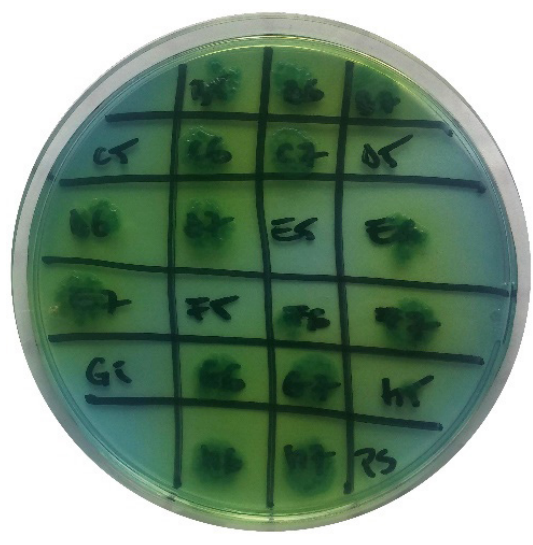

1.B

Figure 1. Representative image for MIC (1.A) and MBC (1.B) determination in Pseudomonas aeruginosa.

(1.A): Position A11-negative control; A12-positive control; MICs were read at positions B6 (EG), C7 (tBA), D7 (nBA), E7 (iBA), F7 (nPA), G7 (iPA), H7 (EA). (1.B): MBCs were read at positions >B5 (EG), C5 (tBA), D5 (nBA), E5 (iBA), F5 (nPA), G5 (iPA), > H5 (EA).

resistance (MRSA vs MSSA) or high environmental adaptability (P. aeruginosa).

All seven alcohols (EA, $n \mathrm{PA}, i \mathrm{PA}, n \mathrm{BA}$, $i \mathrm{BA}, t \mathrm{BA}$ and $\mathrm{EG})$ presented good efficacy at lower concentrations than those used in clinical practice as antiseptics or disinfectants (Table 2). Overall, the best inhibitory effect was noticed for nPA, followed by iPA, nBA and iBA at equal position. tBA presented approximately the same efficacy as EA, though better against E. coli. EG showed the worst efficacy on all bacteria.

All alcohols presented better efficacy on Gram-negative bacteria than on the Gram-positive ones. Notably, the bactericidal and inhibitory effect of all alcohols (except EG) was expressed at similar concentrations in E. coli, but not in $K$. pneumoniae and $P$. aeruginosa, where the $\mathrm{MBC}$ was approximatively four times higher than MIC, and to a concentration level similar to that encountered for most of the Gram-positive bacteria. The highest difference between MIC and MBC was present in P. aeruginosa, especial- ly for EG and EA. Among Gram-positive cocci, E. faecalis presented the lowest susceptibility to alcohols.

\section{Discussions}

By searching the PubMed database for the terms ("antibacterial" AND "alcohol"), more than 37.000 results were displayed, starting from 1945 , peaking in the 1970 s and decreasing until 2000s. Nowadays, the trend for publications regarding the synthesis of new alcohol-based compounds is increasing again. Many studies assessed the antibacterial effects of several chemical agents, and their results regarding EA effect was similar to ours $(10,11)$. Nevertheless, not many studies are performed on antimicrobial effect of pure and common alcohols and their isomers. Only a few books describe their action mechanisms. It is claimed that short-chain alcohols with good water solubility are more suitable as disinfectants, especially when diluted in wa- 
Table 2 - Heat map of inhibitory and bactericidal concentrations of the tested alcohols over the bacterial strains

\begin{tabular}{|c|c|c|c|c|c|c|c|c|c|}
\hline & & & $\mathrm{EA}$ & $\mathrm{nPA}$ & iPA & $\mathrm{nBA}$ & iBA & tBA & EG \\
\hline \multirow{6}{*}{ 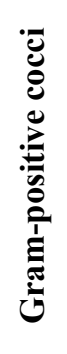 } & \multirow{2}{*}{ MSSA } & MIC & $6.25 \%$ & $3.12 \%$ & $6.25 \%$ & $6.25 \%$ & $6.25 \%$ & $6.25 \%$ & $12.5 \%$ \\
\hline & & $\mathrm{MBC}$ & $25 \%$ & $3.12 \%$ & $6.25 \%$ & $6.25 \%$ & $6.25 \%$ & $25 \%$ & $12.5 \%$ \\
\hline & \multirow{2}{*}{ MRSA } & MIC & $6.25 \%$ & $6.25 \%$ & $6.25 \%$ & $6.25 \%$ & $6.25 \%$ & $12.5 \%$ & $12.5 \%$ \\
\hline & & MBC & $12.5 \%$ & $6.25 \%$ & $6.25 \%$ & $6.25 \%$ & $6.25 \%$ & $12.5 \%$ & $12.5 \%$ \\
\hline & \multirow{2}{*}{ E. faecalis } & MIC & $6.25 \%$ & $6.25 \%$ & $6.25 \%$ & $6.25 \%$ & $6.25 \%$ & $6.25 \%$ & $12.5 \%$ \\
\hline & & $\mathrm{MBC}$ & $25 \%$ & $6.25 \%$ & $6.25 \%$ & $6.25 \%$ & $6.25 \%$ & $25 \%$ & $37.5 \%$ \\
\hline \multirow{6}{*}{ 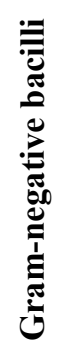 } & \multirow{2}{*}{ E. coli } & MIC & $6.25 \%$ & $3.12 \%$ & $3.12 \%$ & $3.12 \%$ & $3.12 \%$ & $3.12 \%$ & $6.25 \%$ \\
\hline & & MBC & $6.25 \%$ & $3.12 \%$ & $3.12 \%$ & $3.12 \%$ & $3.12 \%$ & $3.12 \%$ & $12.5 \%$ \\
\hline & \multirow{2}{*}{ K. pneumoniae } & MIC & $3.12 \%$ & $3.12 \%$ & $3.12 \%$ & $3.12 \%$ & $3.12 \%$ & $3.12 \%$ & $6.25 \%$ \\
\hline & & MBC & $6.25 \%$ & $6.25 \%$ & $6.25 \%$ & $6.25 \%$ & $6.25 \%$ & $6.25 \%$ & $6.25 \%$ \\
\hline & \multirow{2}{*}{ P. aeruginosa } & MIC & $1.56 \%$ & $1.56 \%$ & $1.56 \%$ & $1.56 \%$ & $1.56 \%$ & $1.56 \%$ & $3.12 \%$ \\
\hline & & $\mathrm{MBC}$ & $>12.5 \%$ & $6.25 \%$ & $6.25 \%$ & $6.25 \%$ & $6.25 \%$ & $6.25 \%$ & $>12.5 \%$ \\
\hline
\end{tabular}

ter, and that the bactericidal effect is different among isomers (the highest activity in n-primary, followed by iso-primary, then secondary and tertiary) (12). Our results are concordant with this hypothesis, $\mathrm{nBA}$ and $\mathrm{iBA}$ presenting better effect than tBA on all bacteria, but nPA a better effect than iPA on MSSA only.

Although several alcohols exhibit antimicrobial activity, only EA, $i$ PA and $n$ PA are used extensively as disinfectant, with the latter two especially in Europe. In general, alcohols show a rapid activity against microorganisms but are not sporicidal; they can inhibit spore germination, but this effect is reversible when the alcohols are evaporated (7).

In Gram-positive bacteria, we found no differences between the MICs and MBCs of primary and iso-structures. Nevertheless, the MICs were higher than in Gram-negative bacteria. This can be explained by the specific cell wall structure of the two bacterial classes (Figure 2).
The specific action mode of alcohols as disinfectants is not so well-understood (7). However, two different mechanisms were proposed: dissolving the lipid membranes and denaturation of proteins.

Biological action of alcohols is a combination of those two different mechanisms (13) and arise from a combination of alcohol-inducing changes in the membrane lipid layer and a specific membrane protein-alcohol interaction (Figure 3). Based on the fact that alcohol efficacy increases in the presence of water (the best efficacy being noticed at a concentration of $60-90 \%$ expressed in volume-to-volume ratio), the first mechanism is thought to dissolve the lipid membrane due to lipophilicity of alcohols, especially those with long alkyl chain $(12,14)$. Therefore, we expected that $n \mathrm{BA}$ be the most effective of studied alcohols, followed by $i \mathrm{BA}$ and PA alcohols.

Alcohols usually alter the lipid composition of cellular membranes, affecting their fluidity. 


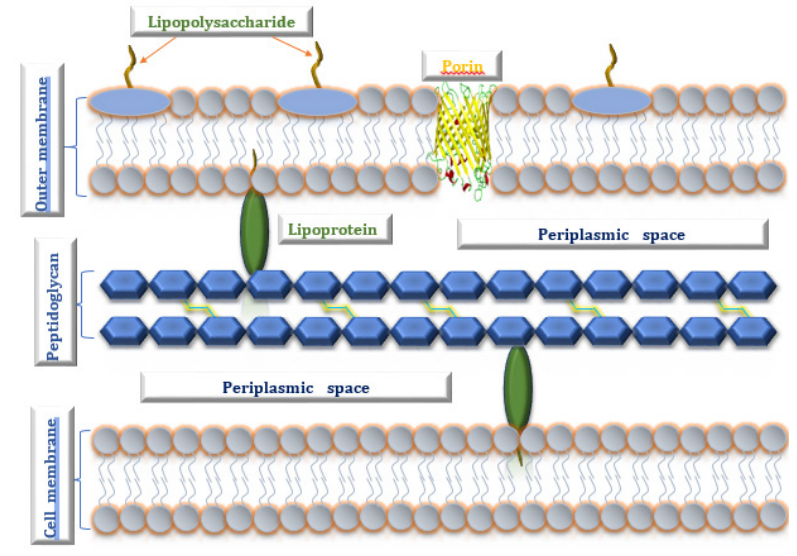

A

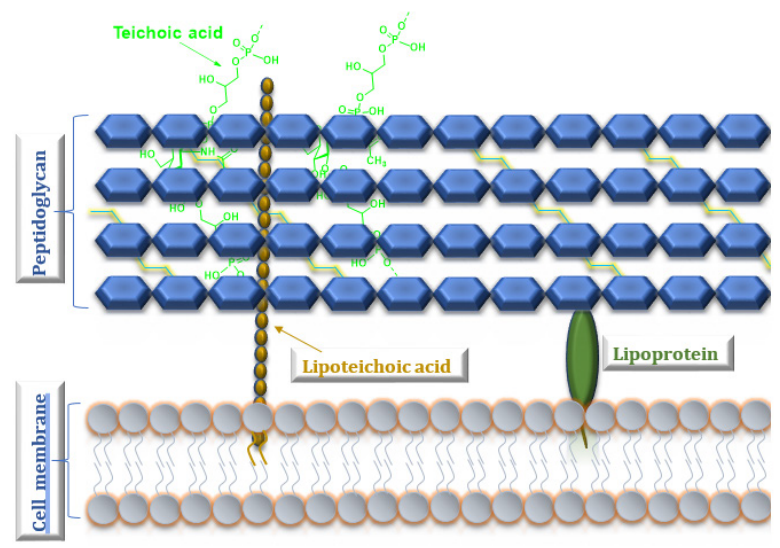

B

Figure 2. (2.A) Gram-negative bacteria present an outer membrane consisting of lipopolysaccharides pierced by porin structures and a thin peptidoglycan layer. (2.B) Gram-positive bacterial wall consists mostly of peptidoglycan pierced by teichoic acid and lipoteichoic acid chains.

As the Gram-negative microorganisms present a thick outer layer of lipopolysaccharide and the inner phospholipidic membrane, it is expected that the alcohols affect this bacterial class efficiently and quickly $(15,16)$. Interestingly, the alcohols inhibited the growth of $K$. pneumoniae, a bacterium that is part of the same family as $E$. coli, in a concentration of about $3 \% \mathrm{~V} / \mathrm{V}$, but the bactericidal effect appeared at higher concentrations. The definitory structure for this bacterial behavior is the polysaccharide capsule, on which the alcohols exert a precipitation effect,

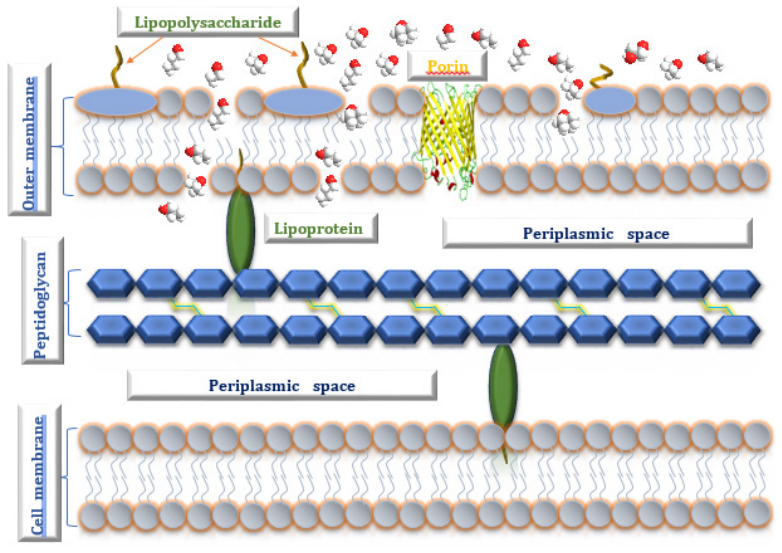

A

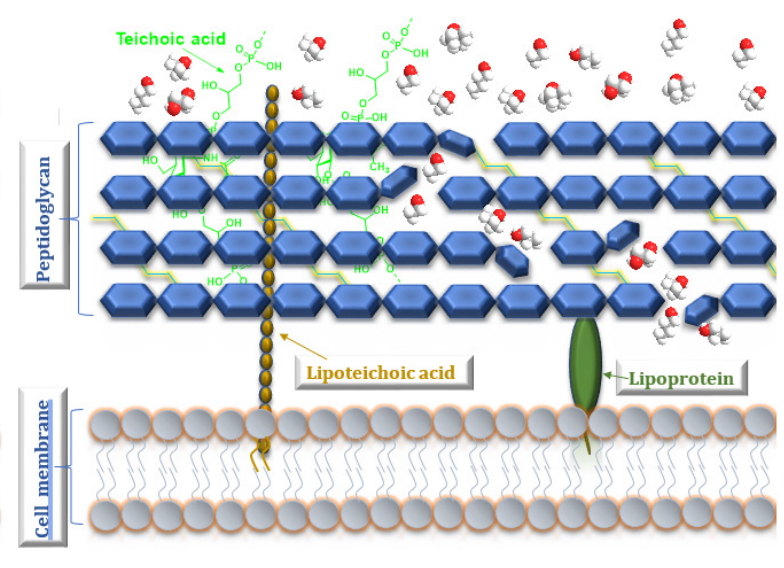

B

Figure 3. (3.A) Disruption of Gram-negative bacterial outer membrane in presence of alcohols. (3.B). Alteration of protein function by alcohols, resulting in peptidoglycan disarrangement in Gram-positive bacteria. 
but the intensity of this effect is dependent on the concentration of the alcohol (17). Moreover, the capsule provides protection against desiccation (18). Many highly adaptable Gram-negative rods such as $P$. aeruginosa can also produce biofilm-associated polysaccharides, becoming part of the most prevalent non-sporulated bacilli in hospital environment (19). As such, though EA inhibited $P$. aeruginosa growth in low concentrations, it could not efficiently kill it. The same is the case of EG, with the supplementary disadvantage of higher MIC and possible kidney, liver or central nervous system toxicity if assimilated (20).

The Gram-positive bacteria present a thick peptidoglycan layer (16) that prevents alcohols to express their lipolytic effect. Nevertheless, the bactericidal effect of alcohols is present, but at higher concentrations. The action mechanism is also different, as the alcohols alter protein function through direct protein interactions and induce cell desiccation (13). These not so efficient action mechanisms of the alcohols may explain the higher but equal MIC and MBC on Gram-positive bacteria. No significant or consistent differences were observed between MSSA and MRSA, but it is known that the effect of alcohols is assigned to specific protein regions of the cell membrane, and is not at all similar to that of the antibiotics that require very specific targets $(13,21)$. Bacteria present specific mechanisms for fighting against antibiotic substances (enzyme production, decreased antibiotic penetration and efflux, changes in target sites or global cell adaptations), but they also present high adaptability to biocide substances, especially if they are in low concentrations (impermeability barrier, efflux pumps, degradation, modification of target or metabolic alterations) $(22,23)$. The eternal fight between new antibiotics and antibiotic resistance is currently shadowing the simplest and most common ways of bacterial persistence in hospital environment due to improper infection control. Alcohols and alcohol formulations are largely used, but some formulations may have better effect and may prevent bacterial adaptation. We show that long-chain alcohols are more efficient against both Gram-positive and Gram-negative bacteria. The drawbacks of long-chain alcohols are related to their smells (powerful) as well as their oily-like consistency. Ethylene glycol should be avoided due to its toxicity hazard and low antimicrobial efficacy. Nevertheless, isopropanol exhibits better properties against bacteria and can be recommend to be used as disinfectant in the detriment of ethanol, the latter having also the disadvantage of high flammability.

As a study limitation, we mention the relatively small number of alcohols and bacterial strains that have been used. Longer-chain alcohols are oily or solid, less soluble in water, making them inadequate for antimicrobial susceptibility testing by microdilution method. We also considered using only prototype strains, which are representative for most bacterial types.

\section{Conclusions}

All alcohols present good effect on bacteria, even in low concentrations. Compared to ethanol as standard, there are better alternatives that can be used as antimicrobials, namely longer-chain alcohols such as propyl or butyric alcohols and their iso- isomers. Bacterial phenotype (highly adaptable bacteria, biofilm formation) and structure (cell wall structure, presence of capsule) may drastically affect the responsiveness to the antimicrobial activity of alcohols, leading to higher bactericidal than inhibitory concentrations.

\section{Acknowledgements}

This work received partial support from the University of Medicine and Pharmacy of Tîrgu Mureș through Internal Grant no. 4/23.12.2014. 
The first two authors share the same contributions to the study design, experimental part, and manuscript preparation.
Abbreviations
EA - ethyl alcohol
EG - ethylene glycol
iBA - iso-butyl alcohol
iPA - iso-propyl alcohol
nBA - n-butyl alcohol
nPA - n-propyl alcohol
tBA - tert-butyl alcohol
$\mathrm{MBC}$ - minimum bactericidal concentration
$\mathrm{MIC}$ - minimum inhibitory concentration

\section{References}

1. Kampf G, Löffler H, Gastmeier P. Hand Hygiene for the Prevention of Nosocomial Infections. Dtsch Ärztebl Int. 2009 Oct;106(40):649-55.

2. Mathur P. Hand hygiene: Back to the basics of infection control. Indian J Med Res. 2011 Nov;134(5):61120. DOI: $10.4103 / 0971-5916.90985$

3. AFE14 - 148to158.pdf[Internet]. Available from: http:// www.who.int/water_sanitation_health/medicalwaste/ 148to158.pdf

4. Tutelcă A, Licker M, Dan L, Orb C, Moldovan R. Nosocomial infections caused by two strains of Klebsiella pneumoniae with different colonial morphotypes and resistance phenotypes isolated from the same sample. Rev Romana Med Lab. 2006;2(1):19-31.

5. Shin J-H, Yue Y, Duan D. Recombinant adeno-associated viral vector production and purification. Methods Mol Biol Clifton NJ. 2012;798:267-84. DOI: 10.1007/978-1-61779-343-1_15

6. Pfäfflin F, Tufa TB, Getachew M, Nigussie T, Schönfeld A, Häussinger D, et al. Implementation of the WHO multimodal Hand Hygiene Improvement Strategy in a University Hospital in Central Ethiopia. Antimicrob Resist Infect Control. 2017;6:3. DOI: 10.1186/s13756016-0165-9

7. McDonnell G, Russell AD. Antiseptics and Disinfectants: Activity, Action, and Resistance. Clin Microbiol Rev. 1999 Jan;12(1):147-79.

8. Girou E, Loyeau S, Legrand P, Oppein F, Brun-Buis- son C. Efficacy of handrubbing with alcohol based solution versus standard handwashing with antiseptic soap: randomised clinical trial. BMJ. 2002 Aug 17;325(7360):362. DOI: 10.1136/bmj.325.7360.362

9. Kampf G, Ostermeyer C. Small volumes of n-propanol (60\%) applied for 3 minutes may be ineffective for surgical hand disinfection. Antimicrob Resist Infect Control. 2014 Apr 24;3:15. DOI: 10.1186/2047-2994-3-15

10. Masaadeh HA, Jaran AS. Determination of the Antibacterial Efficacy of Common Chemical Agents in Cleaning and Disinfection in Hospitals of North Jordan. Am J Appl Sci. 2009 May 31;6(5):811-5. DOI: 10.3844/ ajassp.2009.811.815

11. Mazzola PG, Jozala AF, Novaes LC de L, Moriel P, Penna TCV. Minimal inhibitory concentration (MIC) determination of disinfectant and/or sterilizing agents. Braz J Pharm Sci. 2009 Jun;45(2):241-8. DOI: 10.1590/S1984-82502009000200008

12. Lorian V. Antibiotics in Laboratory Medicine. Lippincott Williams \& Wilkins; 2005. 922 p.

13. Ingólfsson HI, Andersen OS. Alcohol's Effects on Lipid Bilayer Properties. Biophys J. 2011 Aug 17;101(4):84755. DOI: 10.1016/j.bpj.2011.07.013

14. Chatterjee I, Somerville GA, Heilmann C, Sahl H-G, Maurer HH, Herrmann M. Very low ethanol concentrations affect the viability and growth recovery in post-stationary-phase Staphylococcus aureus populations. Appl Environ Microbiol. 2006 Apr;72(4):262736.DOI: 10.1128/AEM.72.4.2627-2636.2006

15. Huffer S, Clark ME, Ning JC, Blanch HW, Clark DS. Role of Alcohols in Growth, Lipid Composition, and Membrane Fluidity of Yeasts, Bacteria, and Archaea. Appl Environ Microbiol. 2011 Sep;77(18):64008. DOI: 10.1128/AEM.00694-11

16. Brown L, Wolf JM, Prados-Rosales R, Casadevall A. Through the wall: extracellular vesicles in Gram-positive bacteria, mycobacteria and fungi. Nat Rev Microbiol. 2015 Oct 1;13(10):620-30. DOI: 10.1038/nrmicro3480

17. Xu J, Yue R-Q, Liu J, Ho H-M, Yi T, Chen H-B, et al. Structural diversity requires individual optimization of ethanol concentration in polysaccharide precipitation. Int J Biol Macromol. 2014 Jun;67:205-9. DOI: 10.1016/j.ijbiomac.2014.03.036

18. Schembri MA, Blom J, Krogfelt KA, Klemm P. Capsule and Fimbria Interaction in Klebsiella pneumo- 
niae. Infect Immun. 2005 Aug;73(8):4626-33. DOI: 10.1128/IAI.73.8.4626-4633.2005

19. Hilliam Y, Moore MP, Lamont IL, Bilton D, Haworth CS, Foweraker J, et al. Pseudomonas aeruginosa adaptation and diversification in the non-cystic fibrosis bronchiectasis lung. Eur Respir J. 2017 Apr;49(4). DOI: 10.1183/13993003.02108-2016

20. Toth-Manikowski SM, Menn-Josephy H, Bhatia J. A Case of Chronic Ethylene Glycol Intoxication Presenting without Classic Metabolic Derangements. Case Rep Nephrol. 2014 Aug 21;2014:e128145.

21. Kohanski MA, Dwyer DJ, Collins JJ. How antibiotics kill bacteria: from targets to networks. Nat Rev Microbiol. 2010 Jun;8(6):423-35. DOI: 10.1038/nrmicro2333

22. Munita JM, Arias CA. Mechanisms of Antibiotic Resistance. Microbiol Spectr [Internet]. 2016 Apr;4(2). Available from: http://www.ncbi.nlm.nih.gov/pmc/ articles/PMC4888801/ DOI: 10.1128/microbiolspec. VMBF-0016-2015

23. Maillard J-Y. Antimicrobial biocides in the healthcare environment: efficacy, usage, policies, and perceived problems. Ther Clin Risk Manag. 2005 Dec;1(4):30720 . 